African Crop Science Journal by African Crop Science Society is licensed under a Creative Commons Attribution 3.0 Uganda License. Based on a work at www.ajol.info/ and www.bioline.org.br/cs DOI: http://dx.doi.org/10.4314/acsj.v23i4.3

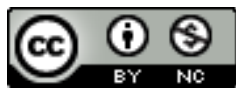

\title{
EXTENT AND PATTERN OF GENETIC DIVERSITY IN ETHIOPIAN WHITE LUPIN LANDRACES FOR AGRONOMICAL AND PHENOLOGICAL TRAITS
}

\author{
MULUGETA ATNAF ${ }^{1,2}$, KASSAHUN TESFAYE $^{2}$, KIFLE DAGNE $^{2}$ and DAGNE WEGARI ${ }^{3}$ \\ ${ }^{1}$ Ethiopian Institute of Agricultural Research, Pawe Research Center, P. O. Box 25, Pawe, Ethiopia \\ ${ }^{2}$ Addis Ababa University, College of Natural Sciences, Department of Microbial, Cellular and Molecular \\ Biology, P. O. Box 1176, Addis Ababa, Ethiopia \\ ${ }^{3}$ CIMMYT-Ethiopia, ILRI Campus, CMC Road, P. O. Box 5689, Addis Ababa, Ethiopia \\ Corresponding author: atnafmulugeta@gmail.com
}

(Received 4 July, 2015; accepted 29 September, 2015)

\begin{abstract}
White lupin (Lupinus albus) is one of four economically important species of the Lupinus genus, and has been traditionally cultivated for thousands of years along the Nile valley, including in Ethiopia. An experiment comprising of 143 Ethiopian White lupin landraces and one genotype from Germany, was undertaken at Merawi in Ethiopia. The objective of the study was to cluster the Ethiopian white lupin accessions into similarity groups and assess the extent and pattern of diversity of the accessions. Data on 10 quantitative agronomic traits were recorded. Landraces significantly differed in most of the traits studied, and a significant number of local accessions performed as high as 5 metric tonnes per hectare of grain yield. Cluster analysis showed that landraces were grouped into seventeen clusters of different sizes, of which five were singletons. Some landraces were grouped together regardless of their geographic origin. On the other hand, landraces from Awi, South Gondar and West Gojam in Ethiopia were distributed over many clusters. Hence, the result did not support a definite relationship between geographic diversity and genetic diversity. Genetic distances between many pairs of clusters were significant, justifying crosses between parents from them to be desirable genetic recombinations and, hence, transgressive segregants.
\end{abstract}

Key Words: Ethiopia, landrace populations, Lupinus albus

RÉSUMÉ

Le lupin blanc (Lupinus albus) est l'une des quatre espèces d'importance économique du genre Lupinus, cette espèce a été traditionnellement cultivée pendant des milliers d'années aux environs de la vallée du Nil, mais aussi en Ethiopie. Une expérimentation comprenant 143 cultivars traditionnels de lupin blanc d'origine Ethiopienne une accession d'origine allemande, a été conduite à Merawi en Ethiopie. L'objectif était de rassembler les accessions d'origine Ethiopiennes au sein des groupes de similarité et d'évaluer l'étendue et la structure de diversité de ces accessions. Des données sur 10 traits agronomiques ont été collectées. Les cultivars ont montré des différences significatives dans la plupart des traits étudiés et un nombre important d'accessions ont eu des rendements impressionnants allant jusqu'à 5 tonnes de grains par hectare. La classification numérique a rassemblé les accessions au sein de dix-sept groups de d'envergures différentes, dont cinq singletons. Certains cultivars ont été groupées ensemble indépendamment de de leur origine géographique. Par ailleurs, les accessions provenant de Awi, Gondar sud et Gojam oust en Ethiopie se sont disperses dans plusieurs groups différents. D'où, le résultat de l'étude n'a pas supporté de façon definitive la thèse de relation entre l'origine géographique et la diversité génétique. Les distances génétiques étaient différentes entre plusieurs paires de groupes, justifiant ainsi que les croisements entre parents sont des désirables de recombinaisons génétiques, et donc ségrégants transgressifs.

Mots Clés: Ethiopie, des populations naturelles, Lupinus albus 


\section{INTRODUCTION}

White lupin (Lupinus albus L., Fabaceae) is one of four economically important species of the Lupinus genus. It consists of over 300 annual species (Hondelmann, 1984). The other three agriculturally important species of the genus are Lupinus angustifolius, Lupinus luteus and Lupinus mutabilis. Molecular evolution studies suggest that three of the four economic species originated from the Mediterranean, and eastern and northern Africa regions; while the fourth important species, Lupinus mutabilis originated from the New World (Wolko et al., 2011). Lupins have an ancient history in agriculture, that trace back to more than 4000 years (Kurlovich, 2002). Though, its domestication first occurred in the Mediterranean and eastern Africa, a real breakthrough that made lupin a modern agricultural crop occurred in Australia and Europe (Clements et al., 2005a).

White lupin $(2 n=4 x=50)$ is a widely known, commercially important, large seeded, annual species. It is a promising annual legume crop for human consumption, green manuring and forage. It has also substantial human nutrition and health importance (Hall, 2005; Lgari et al., 2005; Johnson et al., 2006).

White lupin has been traditionally cultivated for thousands of years along the Nile valley, including in Ethiopia (Kurlovich, 2012). It is locally known in Ethiopia as 'Gibto', and is mainly produced by small holder subsistence farmers around Lake Tana. According to the Ethiopian Central Statistical Agency (ECSA) (2013) report, 107,379 farmers cultivated lupin on a total area of 33,170.03 hectare in 2013 main cropping season. However, farmers' production efforts have not yet been supported by research and/or technology interventions (Yehyis et al., 2010; Atnaf et al., 2015).

Knowledge of the genetic variation between and within populations is an important step for every management strategy directed towards the improvement and conservation of these populations (Xiao et al., 2008). About 300 white lupin landrace accessions have been collected mainly from North Western Ethiopia, including Gojam and Gondar, and have been conserved at the Institute of Biodiversity Conservation of
Ethiopia. With the exception of some passport data, these accessions have never been phenotyped and characterised for important agronomic and phenological traits including grain yield.

Multivariate analyses are useful approaches to characterising populations such as for White lupin, as it considers several agronomic parameters or traits simultaneously. Clustering takes a set of units into account to group them based on their observed characteristics. Principal components analysis is aimed at reducing the dimensionality. That is, it aims to find a smaller number of dimensions (usually 2 or 3 ) that exhibit most of the variation present in the data. This can help to identify the relative importance of individual traits. The objective of this study was to cluster the Ethiopian white lupin accessions into similarity groups and assess the extent and pattern of diversity of the accessions.

\section{MATERIALS AND METHODS}

One hundred forty three Ethiopian white lupin landraces received from the Biodiversity Conservation Institute (IBC) of Ethiopia, plus one sweet genotype from Germany, were used in this study. The landraces considered represent almost $50 \%$ of the total collections at IBC, Ethiopia. The collections were mainly from North Western Ethiopia, including Gojam and Gondar. There were few landrace accessions from South and North Ethiopia. Detailed description of the landraces are presented in Table 1. The landraces were phenotyped at Merawi $\left(11^{\circ} 42^{\prime} \mathrm{N}, 37^{\circ} 17^{\prime} \mathrm{E}\right)$ during 2013/2014 season, with supplemental irrigation. Merawi is located at 1,960 meters above sea level, and receives $1576.55 \mathrm{~mm}$ of rainfall per annum. Its soil is a nitosol with a $\mathrm{pH}$ range of 4.8 - 5.5 (Yihenew, 2002).

The trial was laid down in a $12 \times 12$ simple lattice design. A plot consisted of two rows, 2.5 meter long, with a spacing of $75 \mathrm{~cm}$ between rows and $25 \mathrm{~cm}$ between plants was used. Agronomic and plant protection practices were applied uniformly across plots for the duration of the experiment.

Grain yield was collected per plot and later converted to metric tonnes per hectare. Then, 100 seed weight, number of days from emergence 
TABLE 1. Ethiopian white lupin landraces considered for the study

\begin{tabular}{|c|c|c|c|c|c|c|c|c|c|}
\hline ACC no & IBC code & Zone & District & Altitude & ACC no & IBC code & Zone & District & Altitude \\
\hline Acc1 & 242279 & Awi & Ankesha & 2310 & Acc37 & 238993 & $\mathrm{BD} S p$ & Bahir Dar & 1990 \\
\hline Acc2 & 242280 & Awi & Ankesha & 2185 & Acc38 & 238994 & $\mathrm{BD} \mathrm{Sp}$ & Bahir Dar & 2020 \\
\hline Acc3 & 242281 & Awi & Ankesha & 2310 & Acc39 & 239011 & BD Sp & Bahir Dar & 2090 \\
\hline Acc4 & 242282 & Awi & Ankesha & 2410 & $\operatorname{Acc} 40$ & 239020 & BD Sp & Bahir Dar & 1940 \\
\hline Acc5 & 242266 & WG & Dembecha & 2110 & Acc41 & 239022 & BD Sp & Bahir Dar & 1930 \\
\hline Acc6 & 239044 & Awi & Banja & 2600 & Acc42 & 239023 & BD Sp & Bahir Dar & 1930 \\
\hline Acc7 & 242277 & Awi & Banja & 2560 & Acc43 & 228519 & $S G$ & Dera & \\
\hline Acc8 & 242278 & Awi & Banja & 2560 & Acc44 & 242311 & $S G$ & Dera & 1860 \\
\hline Acc9 & 242283 & Awi & Banja & 2160 & Acc 45 & 242312 & SG & Dera & 1960 \\
\hline Acc10 & 242284 & Awi & Banja & 1960 & Acc46 & 242313 & $S G$ & Dera & 1960 \\
\hline Acc11 & 236619 & Awi & Banja & 2570 & Acc47 & 242314 & $S G$ & Dera & 2160 \\
\hline Acc12 & 239045 & Awi & Banja & 2600 & Acc48 & 242315 & SG & Dera & 2380 \\
\hline Acc13 & 242273 & Awi & Banja & 2490 & Acc49 & 242316 & $S G$ & Dera & 2460 \\
\hline Acc14 & 242274 & Awi & Banja & 2450 & Acc50 & 242268 & WG & Dembecha & 2010 \\
\hline Acc15 & 242276 & Awi & Banja & 2590 & Acc51 & 239018 & WG & $\mathrm{BD} Z$ & 1950 \\
\hline Acc16 & 105018 & & & & Acc52 & 242319 & $S G$ & Dera & 2510 \\
\hline Acc17 & 105005 & Awi & Dangila & 1940 & Acc53 & 105002 & $S G$ & Este & 2420 \\
\hline Acc18 & 228520 & Awi & Dangila & & Acc54 & 226034 & $S G$ & Este & 2560 \\
\hline Acc19 & 242290 & Awi & Dangila & 2240 & Acc55 & 242321 & $S G$ & Este & 2630 \\
\hline Acc20 & 242291 & Awi & Dangila & 2160 & Acc56 & 242219 & $S G$ & Farta & 2280 \\
\hline Acc21 & 242292 & Awi & Dangila & 2060 & Acc57 & 242322 & $S G$ & Farta & 2850 \\
\hline Acc22 & 242293 & Awi & Dangila & 2100 & Acc58 & 242323 & SG & Farta & 2760 \\
\hline Acc23 & 242294 & Awi & Dangila & 2060 & Acc59 & 212754 & $S G$ & Fogera & 1950 \\
\hline Acc24 & 236617 & Awi & Dangila & 2040 & Acc60 & 239008 & WG & Achefer & 2070 \\
\hline Acc25 & 239003 & Awi & Dangila & 2190 & Acc61 & 239029 & WG & Achefer & 2030 \\
\hline Acc26 & 239004 & Awi & Dangila & 2220 & Acc62 & 239033 & WG & Achefer & 2000 \\
\hline Acc27 & 239005 & Awi & Dangila & 2360 & Acc63 & 239038 & WG & Achefer & 2150 \\
\hline Acc28 & 242253 & $E G$ & Machakel & 2140 & Acc64 & 242295 & WG & Achefer & 2050 \\
\hline Acc29 & 239007 & Awi & Dangila & 2190 & Acc65 & 242296 & WG & Achefer & 1975 \\
\hline Acc30 & 242287 & Awi & Fageta & 2550 & Acc66 & 242297 & WG & Achefer & 2010 \\
\hline Acc31 & 242288 & Awi & Fageta & 2425 & Acc67 & 242298 & WG & Achefer & 1990 \\
\hline Acc32 & 239017 & $S G$ & Dera & 2130 & Acc68 & 242299 & WG & Achefer & 2000 \\
\hline Acc33 & 242254 & $E G$ & Machakel & 2150 & Acc69 & 242300 & WG & Achefer & 2060 \\
\hline Acc34 & 242286 & Awi & Guangua & 1740 & Acc70 & 242301 & WG & Achefer & 2090 \\
\hline Acc35 & 105003 & BD Sp & Bahir Dar & 1790 & Acc71 & 242302 & WG & Achefer & 2000 \\
\hline Acc36 & 239021 & $\mathrm{BD} S p$ & Bahir Dar & 1940 & Acc72 & 239009 & WG & Achefer & 2000 \\
\hline Acc73 & 239027 & WG & Achefer & 2060 & Acc109 & 242272 & WG & Bure W & 2500 \\
\hline Acc74 & 239030 & WG & Achefer & 2010 & Acc110 & 105007 & $E G$ & Guzamn & 2430 \\
\hline Acc75 & 239032 & WG & Achefer & 2000 & Acc111 & 216013 & $E G$ & Guzamn & 2500 \\
\hline Acc76 & 239034 & WG & Achefer & 2020 & Acc112 & 239028 & $E G$ & Achefer & 2060 \\
\hline Acc77 & 242308 & WG & $\mathrm{Bd} \mathrm{z}$ & 1975 & Acc113 & 242248 & $E G$ & Guzamn & 2450 \\
\hline Acc78 & 242309 & WG & $\mathrm{Bdz}$ & 2000 & Acc114 & 242252 & $E G$ & Guzamn & 2350 \\
\hline Acc79 & 242310 & WG & $\mathrm{Bdz}$ & 1880 & Acc115 & 105008 & $E G$ & Machakel & \\
\hline Acc80 & 239015 & WG & $\mathrm{Bdz}$ & 1910 & Acc116 & 105009 & $E G$ & Machakel & \\
\hline Acc81 & 239016 & WG & $\mathrm{Bd} \mathrm{z}$ & 1920 & Acc117 & 105010 & $E G$ & Machakel & \\
\hline Acc82 & 239019 & WG & $\mathrm{Bd} \mathrm{z}$ & 2000 & Acc118 & 105011 & $\mathrm{EG}$ & Machakel & \\
\hline Acc83 & 239046 & WG & Bure w & 2520 & Acc119 & 238996 & BD $S$ & Bahir Dar & 2050 \\
\hline Acc84 & 239051 & WG & Bure w & 2120 & Acc120 & 239035 & WG & Achefer & 2050 \\
\hline Acc85 & 236620 & WG & Damot & 2110 & Acc121 & 239002 & WG & Merawi & 2070 \\
\hline Acc86 & 105006 & WG & Dembecha & 2430 & Acc122 & 105015 & $E G$ & Machakel & \\
\hline
\end{tabular}


TABLE 1. Contd.

\begin{tabular}{|c|c|c|c|c|c|c|c|c|c|}
\hline ACC no & IBC code & Zone & District & Altitude & ACC no & IBC code & Zone & District & Altitude \\
\hline Acc87 & 242263 & WG & Dembecha & 2380 & Acc123 & 105016 & $E G$ & Machakel & \\
\hline Acc88 & 242264 & WG & Dembecha & 2430 & Acc124 & 105017 & $E G$ & Machakel & \\
\hline Acc89 & 242265 & WG & Dembecha & 2450 & Acc125 & 242255 & $E G$ & Machakel & 2200 \\
\hline Acc90 & 242267 & WG & Dembecha & 2060 & Acc126 & 242256 & $E G$ & Machakel & 2200 \\
\hline Acc91 & 242269 & WG & Dembecha & 2010 & Acc127 & 242257 & $E G$ & Machakel & 2120 \\
\hline Acc92 & 242270 & WG & Dembecha & 2050 & Acc128 & 242258 & $E G$ & Machakel & 2300 \\
\hline Acc93 & 105001 & WG & Jabi & 2280 & Acc129 & 242260 & $E G$ & Machakel & 2400 \\
\hline Acc94 & 242303 & WG & Mecha & 1950 & Acc130 & 105004 & $N G$ & Belesa & 1820 \\
\hline Acc95 & 242304 & WG & Mecha & 1950 & Acc131 & 239012 & $N G$ & G zuria & 1930 \\
\hline Acc96 & 242305 & WG & Mecha & 2000 & Acc132 & 208464 & Awi & Dangela & 2100 \\
\hline Acc97 & 242306 & WG & Mecha & 2010 & Acc133 & 239060 & $N G$ & G zuria & 1900 \\
\hline Acc98 & 242307 & WG & Mecha & 2010 & Acc134 & 208365 & GUR & Gumer & \\
\hline Acc99 & 236615 & WG & Mecha & 2000 & Acc135 & 225802 & NO & Dermalo & 2800 \\
\hline Acc100 & 236616 & WG & Mecha & 2060 & Acc136 & 242320 & NO & Dermalo & 2800 \\
\hline Acc101 & 238997 & WG & Mecha & 2060 & Acc137 & 207912 & Mk & Adwa & \\
\hline Acc102 & 238999 & WG & Mecha & 2050 & Acc138 & Local & WG & Dembecha & \\
\hline Acc103 & 239001 & WG & Mecha & 2050 & Acc139 & Local & Awi & Fageta & \\
\hline Acc104 & 239010 & WG & Mecha & 2050 & Acc140 & Local & WG & Achefer & \\
\hline Acc105 & 242249 & $E G$ & Baso & 2300 & Acc141 & Local & WG & Mecha & \\
\hline Acc106 & 242250 & $E G$ & Baso & 2310 & Acc142 & Local & SG & Dera & \\
\hline Acc107 & 242251 & $E G$ & Baso & 2300 & Acc143 & Local & $\mathrm{BD} S$ & $\mathrm{Bd} \mathrm{z}$ & \\
\hline Acc108 & 242271 & WG & Bure w & 2450 & Acc144 & Sweet & $\mathrm{Gm}$ & $\mathrm{Gm}$ & \\
\hline
\end{tabular}

Acc no=Accession number; IBC=Institute of Biodiversity \& conservation; WG=West Gojam; EG=East Gojam; BD S= Bahir Dar Special; SG=South Gondar; Fageta=Fageta Lekoma. WG=West Gojam; EG=East Gojam; Bd Z= Bahir Dar Zuria; Jabi=Jabi Tehnan; Baso=Baso Liben; Bure W=Bure Womberema; BD S= Bahir Dar Special; NG=North Gondar; NO=North Omo; GUR=Gurage; MK=Mehakelegnaw; South Gondar; $\mathrm{Gm}=$ Germany; G Zur= Gondar Zuria; Fageta=Fageta Lekoma

to $50 \%$ flowering and $75 \%$ physiological maturity were also determined on plot basis. Number of pods per plant and seeds per pod, plant height, pod length and diameter, and number of branches on main axis were recorded on plant basis. Plant data were assessed on five plants, randomly taken from each plot.

The data were checked for outliers and normality of residuals, using Breeding View of Breeding Management System, before proceeding to analysis (BMS, 2015). Adjusted mean values (best linear unbiased estimators/ BLUE) for all the traits for further analyses were also generated using the same software.

Mean trait data were standardised to mean zero and unity variance in order to minimise biases due to differences in scales of measurement. Multivariate analyses such as Cluster Analysis and Principal Component Analysis, were used. The Principal Components Analyses were meant to identify large contributing traits to the total variation among the populations. Nonhierarchal, and hierarchal clustering of accessions based on the Average Linkage Method were performed using SAS (SAS, 2004), and GenStat software (GenStat, 2013), respectively. Statistics, pseudo $\mathrm{F}$ statistic and pseudo $\mathrm{t}^{2}$ statistic generated by SAS were examined to decide the number of optimum clusters.

Genetic distances between clusters, as standardised Mahalanobis's $\mathrm{D}^{2}$ statistics were calculated as:

$$
D^{2} i j=\left(X_{i}-X_{j}\right) s^{-1}\left(X_{i}-X_{j}\right)
$$

Where $\mathrm{D}^{2}{ }_{\mathrm{ij}}$ is the distance between cases $\mathrm{i}$ and $\mathrm{j}$; $x_{i}$ and $x_{j}$ are the vectors of the values of the variables for cases $\mathrm{i}$ and $\mathrm{j}$; and $\mathrm{s}^{-1}$ is the pooled within groups variance-covariance matrix. 
The $\mathrm{D}^{2}$ values obtained for pairs of clusters were considered as the calculated values of Chisquare $(\chi 2)$ and were tested for significance at $(1$ and $5 \%$ ) probability levels against the tabulated value of $\chi 2$ for ' $\mathrm{P}$ ' degree of freedom, where $\mathrm{P}$ is the number of parameters considered (Singh and Chaudhary, 1985).

Principal components based on correlation matrix, and Euclidian distances were calculated using GenStat software. One of the major reasons that analyses of principal components shall be based on correlation matrix was to standardise each variate (by subtracting its mean and dividing by its standard deviation), which is very useful as the parameters considered in this study did not share a common scale of measurement. Principal components having Eigen value greater than one was considered as significant and presented in the result.

\section{RESULTS}

Landraces were significantly different among themselves for most of the traits studied at genotypic and phenotypic levels (variances not shown). The performance of the landrace accessions phenotyped showed that there was a significant number of white lupin landraces which performed as high as 5 metric tonnes per hectare grain yield (Supplementary Table given). A sweet narrow leafed lupin (Lupinus angustifolius) genotype, introduced from Germany, called Sanabor (Acc144), performed the least (1.01 tonnes per hectare) for grain yield; worse than the local landrace, Acc57 (2.66 tonnes per hectare). The landraces in general were late maturing, i.e. took a mean of 179 days to maturity. The earliest local accession (Acc12) took 168 days to mature, which was still long time. However, Sanabor (Acc144) was the earliest and took 131 days to maturity.

Different lupin diseases such as lupin rust, pleiochaeta root rot, brown leaf spot and phomopsis occurred at different pathogenic level on the local accessions. The severity of rust was scored using 1-9 scale, and some level of variability in resistance/tolerance of the local accessions were observed (data not shown). In general, the local accessions showed moderate resistance to lupin rust. Australian native budworm was observed in the present study at podding stage, on the local accessions.

Cluster analysis. The landraces were grouped into 17 clusters (Table 2 and Fig. 1). Twelve of them comprised of more than one landrace accessions; whereas five clusters were singletons (each containing single accession). The first three clusters contained $100(70 \%)$ accessions out of the total landraces considered. Cluster I contained 78 accessions (54\%) out of 144 ; followed by clusters III, V and II containing 12, 11 , and 10 accessions, in that order. Clusters XIII, $\mathrm{XIV}, \mathrm{XV}, \mathrm{XVI}$, and XVII contained one accession each; whereas the other 8 clusters consisted of accessions ranging from $2-7$.

The local accessions used in this study were originally collected from different regions of Ethiopia, including West and East Gojam, Awi, Bahir Dar Zuria, South and North Gondar, North Omo, Gurage, and Mehakelegnaw (Table 3). However, $95 \%$ of the accessions were from five zones; namely, West Gojam, East Gojam, Awi, South Gondar, and Bahir Dar zuria. Cluster I was mainly (about 90\%) constituted by accessions from three bordering zones of Gojam namely: West Gojam, Awi and Bahir Dar Zuria. Among these, more than $56 \%$ were from west Gojam. Cluster VII consisted of accessions from a single origin, East Gojam. Similarly, cluster X was made up of mainly accessions from South Gondar. The four accessions that did not group and form four separate clusters were Acc10 (Cluster XIII), Acc3 (cluster XIV), Acc116 (cluster XV), and Acc20 (XVI). Three of these accessions (Acc10, Acc3, and Acc20) were collected from the same origin, Awi. The genotype from Germany (Acc144), Sanabor, did not group with any local accessions and was put in a separate cluster, XVII. This genotype is characterised by low grain yield, early flowering and maturity, less number of pods per plant, short, more branches, and is small seeded.

Cluster trait performance is presented in Table 4. Clusters I, II, and III were not significantly divergent. These clusters' accessions were characterised as good yielders, relatively early to flower and mature, large seeded, large number of pods per plant; medium plant height, pod length and diameter, and number of branches on the main axis. Among the singleton clusters, 
TABLE 2. Grouping of the 143 Ethiopian white lupin landraces into different diversity clusters

\begin{tabular}{|c|c|c|c|}
\hline Cluster number & $\begin{array}{c}\text { Number and \% of } \\
\text { accessions }\end{array}$ & Accessions grouped & Origins \\
\hline I & $78(54.17)$ & $\begin{array}{l}\text { Acc141, Acc80, Acc119, Acc99, Acc2, Acc5, Acc112, Acc24, Acc25, Acc61, Acc66, Acc36, } \\
\text { Acc69, Acc48, Acc127, Acc60, Acc114, Acc7, Acc19, Acc63, Acc29, Acc41, Acc101, Acc137, } \\
\text { Acc21, Acc67, Acc109, Acc87, Acc102, Acc42, Acc65, Acc64, Acc82, Acc140, Acc38, Acc76, } \\
\text { Acc97, Acc143, Acc50, Acc85, Acc68, Acc74, Acc79, Acc104, Acc26, Acc35, Acc4, Acc34, } \\
\text { Acc78, Acc37, Acc71, Acc95, Acc47, Acc92, Acc88, Acc44, Acc81, Acc108, Acc40, Acc94, } \\
\text { Acc96, Acc28, Acc9, Acc100, Acc17, Acc51, Acc75, Acc12, Acc27, Acc39, Acc70, Acc86, } \\
\text { Acc14, Acc62, Acc90, Acc11, Acc72, Acc131 }\end{array}$ & $\begin{array}{l}\text { West Gojam, East Gojam, Awi zone, } \\
\text { Bahirdar zuria, South Gondar, North } \\
\text { Gondar, Mehakelegnaw }\end{array}$ \\
\hline II & 10(6.94) & Acc23, Acc59, Acc133, Acc142, Acc111, Acc16, Acc135, Acc93, Acc6, Acc132 & $\begin{array}{l}\text { West Gojam, East Gojam, Awi zone, } \\
\text { South Gondar, North Gondar, North Omo }\end{array}$ \\
\hline III & $11(7.64)$ & Acc22, Acc31, Acc126, Acc33, Acc113, Acc128, Acc125, Acc46, Acc91, Acc32, Acc103 & $\begin{array}{l}\text { West Gojam, East Gojam, Awi zone, } \\
\text { South Gondar }\end{array}$ \\
\hline IV & $7(4.86)$ & Acc117, Acc118, Acc115, Acc54, Acc110, Acc56, Acc122 & East Gojam,South Gondar \\
\hline V & $11(7.64)$ & Acc134, Acc58, Acc52, Acc98, Acc106, Acc15, Acc49, Acc138, Acc139, Acc83, Acc8 & $\begin{array}{l}\text { West Gojam, East Gojam, Awi zone, } \\
\text { South Gondar, Gurage }\end{array}$ \\
\hline VI & $3(2.08)$ & Acc13, Acc89, Acc84 & West Gojam, and Awi zone \\
\hline VII & $3(2.08)$ & Acc123, Acc124, Acc107 & East Gojam \\
\hline VIII & $2(1.39)$ & Acc130, Acc136 & North Gondar and North Omo \\
\hline IX & $3(2.08)$ & Acc120, Acc121, Acc18 & West Gojam, and Awi zone \\
\hline$x$ & $5(3.47)$ & Acc43, Acc57, Acc55, Acc45, Acc73 & South Gondar, and West Gojam \\
\hline$X I$ & $4(2.78)$ & Acc129, Acc30, Acc1, Acc53 & East Gojam, Awi zone, South Gondar \\
\hline XII & $2(1.39)$ & Acc105, Acc77 & West Gojam, \& East Gojam \\
\hline XIII & $1(0.69)$ & Acc10 & Awi Zone \\
\hline XIV & $1(0.69)$ & Acc3 & Awi Zone \\
\hline $\mathrm{XV}$ & $1(0.69)$ & Acc116 & East Gojam \\
\hline $\mathrm{XVI}$ & $1(0.69)$ & Acc20 & Awi Zone \\
\hline XVII & $1(0.69)$ & Acc144 & Germany \\
\hline
\end{tabular}




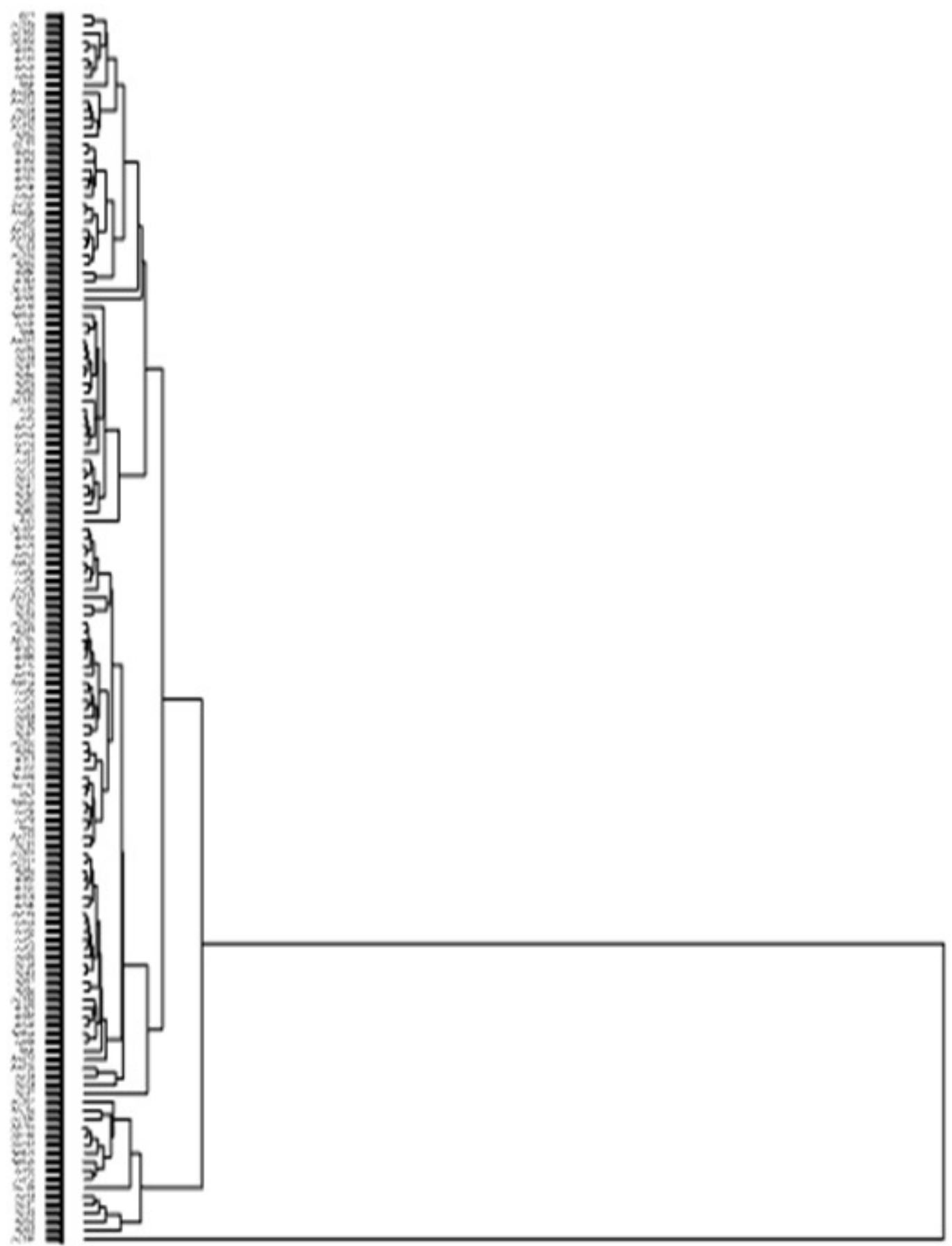

Figure 1. Dendrogram of 143 Ethiopian white lupin accessions and one genotype from Germany based on average linkage hierarchical cluster analysis between groups as represented by Table 2 . 
TABLE 3. Clustering patterns of Ethiopian white lupin landraces from different origins over 17 clusters

\begin{tabular}{|c|c|c|c|c|c|c|c|c|c|c|c|c|c|c|c|c|c|c|}
\hline \multirow[t]{2}{*}{ Origin in Ethiopia } & \multirow{2}{*}{$\begin{array}{l}\text { Number of } \\
\text { Accessions }\end{array}$} & \multicolumn{17}{|c|}{ Number of accessions in each clusters } \\
\hline & & I & II & III & IV & V & VI & VII & VIII & $\mathrm{IX}$ & $x$ & $X I$ & XII & XIII & XIV & $X V$ & $\mathrm{XVI}$ & $X V I I$ \\
\hline West Gojam & 56 & 44 & 1 & 2 & - & 3 & 2 & - & - & 2 & 1 & - & 1 & - & - & - & - & - \\
\hline East Gojam & 21 & 3 & 1 & 5 & 5 & 1 & - & 3 & - & - & - & 1 & 1 & - & - & 1 & - & - \\
\hline Awi zone & 31 & 16 & 3 & 2 & - & 3 & 1 & - & - & 1 & - & 2 & - & 1 & 1 & - & 1 & - \\
\hline Bahir Dar Zuria & 10 & 10 & - & - & - & - & - & - & - & - & - & - & - & - & - & - & - & - \\
\hline South Gondar & 17 & 3 & 2 & 2 & 2 & 3 & - & - & - & - & 4 & 1 & - & - & - & - & - & - \\
\hline North Gondar & 3 & 1 & 1 & - & - & - & - & - & 1 & - & - & - & - & - & - & - & - & - \\
\hline North Omo & 2 & - & 1 & - & - & - & - & - & 1 & - & - & - & - & - & - & - & - & - \\
\hline Gurage & 1 & - & - & - & - & 1 & - & - & - & - & - & - & - & - & - & - & - & - \\
\hline Mehakelegnaw & 1 & 1 & - & - & - & - & - & - & - & - & - & - & - & - & - & - & - & - \\
\hline Unknown & 1 & - & 1 & - & - & - & - & - & - & - & - & - & - & - & - & - & - & - \\
\hline Germany & 1 & - & - & - & - & - & - & - & - & - & - & - & - & - & - & - & - & 1 \\
\hline Total & 144 & 78 & 10 & 11 & 7 & 11 & 3 & 3 & 2 & 3 & 5 & 4 & 2 & 1 & 1 & 1 & 1 & 1 \\
\hline
\end{tabular}


TABLE 4. Cluster mean for 10 characters in Ethiopian white lupin landraces

\begin{tabular}{|c|c|c|c|c|c|c|c|c|c|c|c|c|c|c|c|c|c|c|}
\hline \multirow[t]{2}{*}{ Par } & \multicolumn{18}{|c|}{ Cluster } \\
\hline & I & II & III & IV & V & VI & VII & VIII & IX & $x$ & XI & XII & XIII & XIV & $X V$ & $X V I$ & $X V I I$ & GM \\
\hline DF & 78.76 & 80.12 & 80.10 & 86.05 & 84.77 & 80.52 & 91.30 & 86.46 & 80.00 & 92.17 & 89.74 & 86.99 & 85.18 & 82.56 & 87.49 & 92.64 & 62.95 & 81.2 \\
\hline DM & 176.3 & 180.9 & 180.5 & 188.6 & 182.0 & 177.8 & 189.3 & 190.3 & 175.9 & 187.3 & 183.8 & 176.5 & 181.3 & 173.0 & 192.4 & 177.1 & 131.5 & 178.9 \\
\hline $\mathrm{PH}$ & 133.0 & 124.5 & 133.8 & 129.0 & 137.6 & 123.8 & 126.8 & 146.9 & 129.9 & 124.7 & 132.8 & 119.7 & 129.7 & 123.6 & 128.3 & 136.3 & 48.26 & 131.3 \\
\hline BR & 7.79 & 7.34 & 8.55 & 8.76 & 8.84 & 9.47 & 8.42 & 8.59 & 7.93 & 10.23 & 9.13 & 9.37 & 7.79 & 9.69 & 10.37 & 7.71 & 10.37 & 8.2 \\
\hline PL & 8.82 & 8.90 & 8.45 & 8.22 & 8.79 & 8.86 & 8.18 & 8.60 & 8.67 & 8.55 & 8.70 & 9.02 & 8.66 & 9.01 & 7.31 & 9.31 & 4.91 & 8.7 \\
\hline PD & 6.56 & 6.75 & 6.27 & 6.31 & 6.50 & 6.61 & 6.18 & 6.60 & 6.79 & 6.51 & 6.33 & 6.84 & 7.15 & 6.79 & 5.83 & 7.18 & $* *$ & 6.53 \\
\hline PN & 83.27 & 84.90 & 97.51 & 84.85 & 88.53 & 83.58 & 89.56 & 91.92 & 65.23 & 83.64 & 86.56 & 74.75 & 99.47 & 92.20 & 86.20 & 102.8 & 25.80 & 84.75 \\
\hline SN & 5.44 & 5.26 & 5.25 & 5.14 & 5.30 & 5.07 & 5.00 & 5.60 & 5.23 & 5.08 & 5.40 & 5.25 & 5.80 & 5.70 & 5.20 & 5.40 & 4.30 & 5.35 \\
\hline SW & 32.26 & 31.08 & 30.54 & 28.31 & 31.50 & 32.97 & 25.94 & 29.86 & 35.89 & 30.99 & 29.50 & 35.93 & 32.87 & 33.27 & 24.44 & 34.40 & 17.93 & 31.53 \\
\hline GY & 4.88 & 4.26 & 4.64 & 3.62 & 4.23 & 3.98 & 3.20 & 3.73 & 4.47 & 2.96 & 3.65 & 3.96 & 4.87 & 5.79 & 2.71 & 4.53 & 1.01 & 4.48 \\
\hline
\end{tabular}

Par=Parameter; DF=Days to $50 \%$ flowering; DM=days to $75 \%$ physiological maturity; $\mathrm{PH}=P$ lant height in centimeter; $\mathrm{BR}=$ Number of branches on the main axis; $\mathrm{PL}=\mathrm{Pod}$ length in mil meter; $\mathrm{PD}=$ Pod diameter in mill meter; $\mathrm{PN}=$ Number of pods per plant; $\mathrm{SN}=$ Number of seeds per pod; $\mathrm{SW}=100$ seed weight in gram; $\mathrm{GY}=\mathrm{Grain}$ yield in tones per hectare; $\mathrm{GM}=\mathrm{Grand}$ mean of a given trait/parameter ${ }^{* *}=$ missed value as only single accession (144) with missed value has grouped 
TABLE 5. Intra- (bolded diagonals) and Inter- cluster distance between Ethiopian white lupin landraces categorised into 17 clusters

\begin{tabular}{|c|c|c|c|c|c|c|c|c|c|c|c|c|c|c|c|c|c|}
\hline $\mathrm{CL}$ & I & II & III & IV & V & VI & VII & VIII & IX & $x$ & $X I$ & XII & XIII & XIV & $X V$ & $\mathrm{XVI}$ & XVII \\
\hline I & 1.23 & & & & & & & & & & & & & & & & \\
\hline II & 13.56 & 5.33 & & & & & & & & & & & & & & & \\
\hline III & 11.67 & 13.60 & 5.14 & & & & & & & & & & & & & & \\
\hline IV & $61.47^{\star *}$ & $35.32^{* *}$ & $35.04^{* *}$ & 6.05 & & & & & & & & & & & & & \\
\hline V & $25.22^{\star *}$ & $22.93^{*}$ & 17.11 & $20.46^{*}$ & 5.14 & & & & & & & & & & & & \\
\hline VI & $22.18^{*}$ & $21.10^{*}$ & 16.04 & $42.48^{* *}$ & $20.34^{*}$ & 7.74 & & & & & & & & & & & \\
\hline VII & $128.6^{* *}$ & $83.36^{* *}$ & $87.34^{* *}$ & $20.20^{*}$ & $54.16^{* *}$ & $97.84^{\star *}$ & 7.74 & & & & & & & & & & \\
\hline VIII & $67.95^{* *}$ & $53.28^{* *}$ & $52.90^{* *}$ & $21.32^{*}$ & $21.32^{*}$ & $66.56^{\star *}$ & $46.96^{\star *}$ & 8.55 & & & & & & & & & \\
\hline IX & 12.86 & $26.47^{* *}$ & $29.06^{* *}$ & $62.63^{* *}$ & $25.82^{* *}$ & $21.70^{*}$ & $128.3^{* *}$ & $68.53^{* *}$ & 7.74 & & & & & & & & \\
\hline$X$ & $129.5^{\star *}$ & $97.41^{* *}$ & $96.24^{* *}$ & $31.07^{* *}$ & $45.95^{\star *}$ & $76.02^{\star *}$ & $25.46^{\star *}$ & $46.44^{* *}$ & $107.7^{* *}$ & 6.72 & & & & & & & \\
\hline XI & $70.19^{* *}$ & $53.34^{* *}$ & $52.13^{* *}$ & 17.13 & 14.17 & $50.53^{* *}$ & $24.41^{* *}$ & $19.48^{*}$ & $65.07^{* *}$ & 15.75 & 7.17 & & & & & & \\
\hline XII & $51.86^{* *}$ & $49.06^{* *}$ & $52.61^{* *}$ & $51.46^{* *}$ & $22.01^{*}$ & $26.88^{\star *}$ & $83.05^{\star \star}$ & $62.75^{\star \star}$ & $30.86^{* *}$ & $42.79^{* *}$ & $28.50^{* *}$ & 8.55 & & & & & \\
\hline XIII & $31.73^{\star *}$ & $28.27^{\star *}$ & $30.43^{\star \star}$ & $43.48^{\star \star}$ & $22.79^{*}$ & $51.66^{\star \star}$ & $81.80^{\star \star}$ & $36.74^{\star *}$ & $42.95^{\star *}$ & $85.66^{\star *}$ & $40.00^{\star *}$ & $45.31^{* *}$ & 0.00 & & & & \\
\hline XIV & $31.80^{* *}$ & $60.73^{\star *}$ & $44.39^{\star \star}$ & $102.5^{\star \star}$ & $54.83^{* *}$ & $48.57^{\star \star}$ & $174.1^{* *}$ & $116.8^{* \star}$ & $47.46^{\star *}$ & $151.0^{* *}$ & $95.20^{* *}$ & $59.18^{* *}$ & $48.63^{* *}$ & 0.00 & & & \\
\hline XV & $153.5^{\star \star}$ & $120.7^{* *}$ & $102.3^{\star \star}$ & $33.69^{\star \star}$ & $88.17^{\star *}$ & $107.5^{\star \star}$ & $49.35^{\star *}$ & $65.43^{\star \star}$ & $152.9^{* \star}$ & $56.28^{\star \star}$ & $65.22^{\star *}$ & $129.2^{\star \star}$ & $126.4^{\star \star}$ & $194.2^{\star \star}$ & 0.00 & & \\
\hline XVI & $120.9^{* \star}$ & $108.4^{* \star}$ & $113.5^{\star \star}$ & $105.0^{* \star}$ & $62.66^{\star \star}$ & $121.2^{\star *}$ & $89.37^{\star \star}$ & $87.83^{\star \star}$ & $114.4^{\star \star}$ & $83.35^{\star \star}$ & $53.70^{\star \star}$ & $61.96^{\star *}$ & $64.70^{\star \star}$ & $138.6^{\star \star}$ & $217.2^{\star *}$ & 0.00 & \\
\hline XVII & $1252^{\star \star}$ & $1278^{\star \star}$ & $1213^{\star *}$ & $1341^{* *}$ & $1364^{* \star}$ & $1151^{\star *}$ & $1437^{\star \star}$ & $1563^{\star *}$ & $1270^{\star \star}$ & $1438^{\star \star}$ & $1420^{\star \star}$ & $1324^{\star *}$ & $1494^{\star \star}$ & $1302^{\star \star}$ & $1269^{\star \star}$ & $1697^{\star \star}$ & 0.00 \\
\hline
\end{tabular}

$\mathrm{CL}=$ Clusters; ${ }^{*}$ \& ${ }^{* *}=$ Significant at 0.5 and 0.1 alpha levels, respectively 
TABLE 6. Eigen vectors, explained variance, and Eigen values of the first significant three Principal components for 10 parameters of 144 Ethiopian white lupin landraces

\begin{tabular}{lccc}
\hline Parameter & \multicolumn{3}{c}{ Eigen vectors } \\
\cline { 2 - 4 } & PCA1 & PCA2 & PCA3 \\
\hline Number of branches on the main axis & -0.249 & 0.342 & 0.111 \\
Days to flowering & -0.051 & 0.547 & 0.196 \\
Days to maturity & 0.094 & 0.544 & -0.030 \\
Grain yield & 0.405 & -0.264 & -0.196 \\
Pod diameter & 0.395 & 0.167 & 0.379 \\
Plant height & 0.370 & 0.216 & -0.281 \\
Pod length & 0.424 & 0.055 & 0.269 \\
Number of pods per plant & 0.228 & 0.339 & -0.480 \\
100 seed weight & 0.350 & -0.129 & 0.523 \\
Number of seeds per pod & 0.339 & -0.097 & -0.334 \\
Eigen value & 3.823 & 2.599 & 1.046 \\
Explained variance (\%) & 38.230 & 25.990 & 10.460 \\
Cumulative variance & 38.230 & 64.220 & 74.680 \\
\hline
\end{tabular}

PCA = Principal component analysis

cluster XIV contained an accession (Acc3) with the highest yield and relatively short plant height, large seeded, and large number of pods per plant. On the contrary, cluster XV having Acc116 was characterised by lowest yielder and small seeded. The other singleton cluster XVI contained one accession with the highest number of pods per plant, large seeded and average in grain yield.

Pair-wise generalised squared distances $\left(\mathrm{D}^{2}\right)$ among the seventeen clusters are presented in Table 5. There were 136 possible pair-wise genetic distances between any two clusters. Among these, only 9 genetic distances (between clusters I and II, I and III, I and IX, II and III, III and V, III and VI, IV and XI, V and XI, and X and $\mathrm{XI}$ ) were not significant $(\mathrm{p}>0.05)$. The remaining genetic distances were significant $(\mathrm{p}<0.05)$, to highly significant $(\mathrm{p}<0.01)$. The maximum distance was found between clusters XVI and XVII $\left(D^{2}=\right.$ 1697), and the distances between any cluster and cluster XVII (the cluster containing Sanabor) were maximum and very highly significant $(\mathrm{P}<0.01)$.

Principal component analysis (PCA). The first three principal components were found to be significant (Eigen value greater than 1) and accounted for about $75 \%$ of the total variation
(Table 6). The first PCA component explained $38 \%$ of the total variance, and the first and second PCA components accounted for $64 \%$ of the variation. Parameters that contributed relatively more with an Eigen vector value (0.424 - 0.339) for the first PCA were grain yield, pod length, pod diameter, plant height, 100 seed weight and number of seeds per pod. Thus, this PCA was associated with yield and architectural traits of lupin. Most of the variations accounted to the second PCA were contributed by two phenological traits, days to flowering and maturity; and hence, this PCA was associated with growth duration of the accessions. The third PCA explained about $10.5 \%$ of the variation, and yield component traits such as 100 seed weight and number of pods per plant contributed much of its variation.

\section{DISCUSSION}

Presence of a significant genetic variation among Ethiopian white lupin landraces and the performance of significant number of landraces to levels as high as 5 metric tonnes per hectare grain yield, indicate a huge available genetic potency in terms of grain yield; which could easily be exploited through breeding and selection. A 
similar result was reported by Christiansen et al. (2000), from Egypt, where he showed the importance of landraces for breeding. Another report by Gonzalez-Andres et al. (2007), showed variability of Spanish white lupin local accessions for grain yield and its components. Some level of variability in resistance/tolerance of the local accessions were observed for lupin rust (data not shown). In general, however, the local accessions showed moderate resistance to lupin rust. Occurrences and importance of those white lupin diseases, and resistance breeding achievements have been reported in different parts of the world (Thomas, 2003; Thomas et al., 2008a; Luckett et al., 2009).

The performance of Sanabor (the genotype introduced from Germany) was the poorest (1.01 tonnes per hectare). However, contradictory grain yield performance was reported by Yeheyis et al. (2012) in Ethiopia, whereby Sanabor performed better than our landraces. Nevertheless, these authors considered a few local accessions that could not represent the available huge diversity in the country. In any case, however, Sanabor could not perform comparably for grain yield with those local accessions performed as high as 5 tonnes per hectare.

Presence of highly significant variation in maturity between Sanabor and the Ethiopian landraces suggests that the former could be used as a source of genes for earliness to improve late maturing Ethiopian landraces. However, fertile segregating populations and/or hybrids under natural conditions, from inter-specific crossing between the two populations (landraces from Lupinus albus and Sanabor from Lupinus angustifolius) have hitherto not been reported, except fertile F1 and F2 plants (Kurlovich and Kartuzova, 2002; Clements et al., 2008).

In our study, landraces were grouped into 17 diverse clusters containing significantly different landraces ranging from 1 to 78 . About $90 \%$ of the landraces constituted the Cluster I and were from three bordering zones of Gojam namely: West Gojam, Awi, and Bahir Dar Zuria. This result indicates that there might have been exchange of seeds, and seed trade between farmers, and gene flow across boundaries of those areas (Forsberg et al., 2015). On the other hand, accessions from non-bordering origins were entirely constituted by a particular cluster such as landraces from South Gondar and East Gojam constituent cluster IV, and those from North Gondar and North Omo form cluster VIII. A similar result on local field pea, and faba bean accessions in Ethiopia was reported by Gemechu et al. (2005). One possible reason among others could be that the landraces were introduced from a similar source.

Landraces from the same origin were not all grouped into the same cluster, except accessions from Bahir Dar Zuria in which all the 10 accessions from this zone grouped into Cluster I. This result is in agreement with that of a Moroccan lupin local accessions. The local accessions were clustered regardless of their geographic origin (Sbabou et al., 2010). Distribution of accessions of similar origin into different significantly divergent clusters, might indicate the diversity of accessions within the origin. The distribution of accessions from Awi, East Gojam and West Gojam, over different clusters, was quite high covering 10,9, and 8 clusters, respectively. Moreover, each of the three singleton clusters contained accessions from Awi. This might suggest that accessions from Awi were more diverse than others. The distribution and patterns of accessions, over different clusters from these three major geographic origins, would suggest future collections of local accessions in those geographic regions with particular emphasis on Awi, followed by East Gojam, and West Gojam for future national collection mission in white lupin. Supportive results that Ethiopian accessions formed a very distinct and separate grouping/gene pool than others, was reported from Australia (Raman et al., 2014).

The maximum distance among Ethiopian accessions lies between Clusters XV and XVI $\left(D^{2}=217.23\right)$; followed by distances between clusters XIV and XV $\left(D^{2}=194.18\right)$, and VII and XIV $\left(D^{2}=174.11\right)$. Those cluster pairs that exhibited the first two maximum genetic distances were all singletons. Maximum genetic recombination and variation in the subsequent generation, is expected from crosses that involve parents from the clusters characterised by maximum distances. Thus, it could maximise opportunities for transgressive segregation, since 
a higher probability that unrelated accessions would contribute unique desirable alleles at different loci.

Genetic distance, as good indicator of transgression and heterosis, has been reported by several authors on many crops (Mulugeta et al., 2013; Pickup et al., 2013). Hence, the attempt to cluster Ethiopian white lupin accessions using multivariate analyses, in the present study, is a significant precursor to initiating a white lupin breeding programme. However, the selection of parents for a particular cross should also consider the special advantages of each cluster and accession within a cluster, depending on specific objectives of hybridisation programmes. Members within a cluster are assumed to be more closely related, in terms of trait under consideration than with members in different clusters (Saeed et al., 2008; Million, 2012; Habtamu and Million, 2013).

Principal components analyses in this study showed that the first three PCAs explained about $75 \%$ of the variation. The amount of explained variance by the first PCA and parameters that contributed relatively more, clearly indicated that grain yield and architectural traits of lupin are important traits that could be considered for lupin breeding and selection. Two important phenological traits, for days to flowering and maturity, accounted most of the variations explained by the second PCA. A similar finding on common bean in Ethiopia was reported by Hirpa et al. (2013).

\section{CONCLUSION}

There exists high genetic diversity in the Ethiopian white lupin landraces, and a significant number of landrace accessions yield as high as 5 metric tonnes per hectare of grain. However, the extent and pattern of the existing genetic diversity does not strictly follow the geographic origins. Most of the genetic distances between clusters are significant, suggesting desirable genetic recombination and variation in subsequent generation from crosses that involve parents from those clusters characterised by maximum distances. Thus, this could maximise opportunities for transgressive segregation as there is a higher probability that unrelated accessions would contribute unique desirable alleles at different loci. Hence, the attempt to cluster Ethiopian white lupin landraces using multivariate analyses with the present study is of practical importance to start a white lupin breeding programme. On the other hand, the distribution and pattern of landrace accessions over significantly different clusters from the three major geographic origins would suggest future collections of local accessions in those geographic regions with particular emphasis to East Gojam, followed by Awi and West Gojam in that order of importance for future national collection mission in white lupin.

\section{ACKNOWLEDGEMENT}

The study was part of $\mathrm{PhD}$ research work of the senior author. The financial support of Ethiopian Institute of Agricultural Research (EIAR) and Pawe Agricultural Research Center through Sustainable Intensification of Maize-Legume Systems for Food Security in Eastern and Southern Africa (SIMLESA) project, is appreciated. The local accessions were kindly provided by Institute of Biodiversity Conservation of Ethiopia.

\section{REFERENCES}

Atanf, M., Tesfaye, K. and Kifle, D. 2015. The importance of legumes in the Ethiopian farming system and overall economy: An overview. American Journal of Experimental Agriculture 7(6):347-358.

Christiansen, J.L., Raza, S., Jørnsgård, B., Mahmoud, S.A. and Ortiz, R. 2000. Potential of landrace germplasm for genetic enhancement of white lupin in Egypt. Genetic Resources and Crop Evolution 47: 425-430.

Clements, J.C., Buirchell, B.J., Yang, H., Smith, P.M.C., Sweetinghum, M.W. and Smith, C.G. 2005a. Lupin. Chapter 9. In: Sing, R. and Jauhar, P. (Eds.). Genetic resources, chromosome engineering, and crop improvement: V(1). Grain Legumes. CRC Press.

Clements, J., Prilyuk, L., Quealy, J. and Francis, G. 2008. Inter-specific crossing among the new world species for Lupinus mutabilis crop improvement. In: Lupins for health and 
Wealth. Proceedings of the $12^{\text {th }}$ International Lupin Conference. Palta, J.A. and Brger, J.B. (Eds.). International Lupin Association, Canterbury, New Zealand. pp. 324-327.

Ethiopian Central Statistical Agency (ECSA). 2013. Report on area and production of crops (Private peasant holdings, Meher season). Addis Ababa, Ethiopia.

Forsberg, N.E.G., Russell, J., Macaulay, M., Leino, M.W. and Hagenblad, J. 2015. Farmers without borders - genetic structuring in century old barley (Hordeum vulgare). Heredity 114: 195-206.

Gemechu, K., Mussa, J., Tezera, W. and Getnet, D. 2005. Extent and pattern of genetic diversity for morpho-agronomic traits in Ethiopian highland pulse landraces: I. Field pea (Pisum sativum L.). Genetic Resources and Crop Evolution 52:539-549.

GenStat. 2013. GenStat Statistical software. GenStat for Windows 16th Edition. VSN International, UK.

Gonzalez-Andres, F., Casquero, P.A., San-Pedro, C. and Hernandez-Sanchez, E. 2007. Diversity in white Lupin (Lupinus albus L.) landraces from Northwest Iberian Plateau. Genetic Resources and Evolution 54:27-44.

Habtamu, S. and Million, F. 2013. Multivariate analysis of some Ethiopian field pea (Pisum sativum L.) genotypes. International Journal of Genetics and Molecular Biology 5(6): 78-87.

Hall R.S. 2005. Australian sweet lupin flour addition reduced the glycaemic index of a white bread breakfast without affecting palatability in healthy human volunteers. Asia Pacific Journal of Clinical Nutrition 14: 9197

Hirpa, L., Nigussie, D., Setegn, G., Geremew, B. and Firew, M. 2013. Multivariate analysis as a tool for indirect selection of common bean genotypes (Phaseolus vulgaris L) for soil acidity tolerance under field conditions. Science, Technology and Arts Research Journal 2(2): 07-15.

Hondelmann, W. 1984.The lupin - ancient and modern crop plant. Theoretical and Applied Genetics 68: 1-8.

Johnson, S.K., Chua, V., Hall, R.S. and Baxter, A.L. 2006. Lupin kernel fiber foods improve bowel function and beneficially modify some putative faecal risk factors for colon cancer in men. British Journal of Nutrition 95(2): 372-8.

Kurlovich, B.S. and Kartuzova, L.T. 2002. Lupin breeding. Chapter 13. In: Kurlovich, B.S. (Ed.). Lupins (Geography, classification, genetic resources, and breeding). OY International North Express. St. Petersburg, RussiaPellosniemi, Finland. pp. 351-374.

Kurlovich, B.S. 2002.The history of lupin domestication. Chapter 5. In: Kurlovich, B.S. (Ed.). Lupins (Geography, classification, genetic resources, and breeding). OY International North Express. St. Petersburg, Russia-Pellosniemi, Finland. pp. 147-164.

Lqari, H., Pedroche, J., Girion-Calle, J. and Vioque, J. 2005. Production of lupinus angustifolius protein hydrolysates with improved functional properties. Fats and Oils 56(2): 135-140.

Luckett, D., Cowley, R.B., Richards, M.F. and Roberts, D.M. 2009. Breeding Lupinus albus for resistance to the root pathogen Pleiochaeta setosa. European Journal of Plant Pathology 125(1):131-141.

Million, F. 2012. Variability, heritability and association of some morpho-agronomic traits in field pea (Pisum sativum L.) genotypes. Pakistan Journal of Biological Sciences 15:358-366.

Mulugeta, A., Hussein, M. and Habtamu, Z. 2013. Estimation of better parent and economic heterosis for yield and associated traits in common beans. Journal of Applied Biosciences 71:5706-5714.

Pickup, M., Field, D.L., Rowell, D.M. and Young, A.G. 2013. Source population characteristics affect heterosis following genetic rescue of fragmented plant populations. Proceedings of the Royal society Biological Sciences 280(1750): 2058.

Raman, R., Cowley, R.B., Raman, H. and Luckett, D.J. 2014. Analyses using SSR and DArT molecular markers reveal that Ethiopian accessions of white Lupin (Lupinus albus L.) represent a unique gene pool. Open Journal of Genetics 4: 87-98.

Saeed, 2008. Genetic variability, correlation and path analysis studies in garden pea (Pisum 
sativum L.). Journal of Agricultural Research 46(4):333-340.

SAS. 2004. System Analysis Software. Version 9.1.2. SAS Institute INC. Cary, North Carolina, USA.

Sbabou, L., Brhada, F., Alami, I.T. and Maltouf, A.F. 2010. Genetic diversity of Moroccan Lupinus germplasm investigated using ISSR and AFLP markers. International Journal of Agriculture and Biology 12: 26-32.

Singh, R.K. and Chaudhary, B.D. 1985. Biometrical methods in quantitative genetic analysis. Kalyani Publishers, New Delhi, India.

The IBP Breeding Management System Version 3.0.8; 2015. The Integrated Breeding Platform. https://www.integratedbreeding.net/ breeding-management-system.

Thomas, G. 2003. Lupin anthracnoseidentification and management. Farm note No. 15/2003. Department of Agriculture and Food, Perth, Western Australia.

Thomas, G., Jones, R. and Vanstone, V. 2008a. Diseases of lupin. Chapter 9. In: White, P., French, B. and Mclarty, A. (Eds.). Producing lupins, 2nd Edition. Department of Agriculture and Food, Perth, Western Australia. pp. 101-120.
Wolko, B., Clements, J.C., Naganowska, B., Nelson, M.N. and Yang, H. 2011. Lupinus. In: Kole, C. (Ed.). Wild crop relaives: Genomic and breeding resources. Springer Berlin Heidelberg. pp. 153-206.

Xiao-XuZ, Jian-Ping, G., Shu-Min, W., QingChang, L., Robert, R. and Rebecca, F. 2008. Genetic diversity and core collection of Alien (Pisum sativum L.) Germplasm. Acta Agron Sin. 34(9): 1518-1528.

Yeheyis, L., Kijora, C., Solomon, M., Anteneh, G. and Peters, K.J. 2010. White lupin (Lupinus albus L.), the neglected multipurpose crop: Its production and utilization in the mixed crop-livestock farming system of Ethiopia. Livestock Research for Rural Development. Volume 22, Article \#74. Retrieved March 28, 2015, from http://www.lrrd.org/lrrd22/4/ yehe22074.

Yeheyis, L., Kijora, C., van Santen, E. and Peters, K. 2012. Sweet annual Lupins (Lupinus spp.); Their adaptability and productivity in different agro-ecological zones of Ethiopia. Journal of Animal Science Advances 2(2): 201-215.

Yihenew, G. 2002. Selected chemical and physical characteristics of soils of Adet Research Center and its testing sites in Northwestern Ethiopia. Ethiopian Journal of Natural Resources 4(2):199-215. 